\title{
Une investigation géographique aux marges de l'administration française : les communes de moins de cinquante habitants
}

Jean-Baptiste Grison

\section{(2) OpenEdition}

Journals

Édition électronique

URL : http://journals.openedition.org/cdg/1846

DOI : $10.4000 /$ cdg. 1846

ISSN : 2107-7266

Éditeur

UMR 245 - CESSMA

\section{Référence électronique}

Jean-Baptiste Grison, « Une investigation géographique aux marges de l'administration française : les communes de moins de cinquante habitants ", Carnets de géographes [En ligne], 1 | 2010, mis en ligne le 01 octobre 2010, consulté le 07 mai 2019. URL : http://journals.openedition.org/cdg/1846 ; DOI : $10.4000 /$ cdg. 1846

\section{(c) $($ ) $\odot \odot$}

La revue Carnets de géographes est mise à disposition selon les termes de la Licence Creative Commons Attribution - Pas d'Utilisation Commerciale - Pas de Modification 4.0 International. 


\title{
Une investigation géographique aux marges de l'administration française : les communes de moins de cinquante habitants
}

\author{
Jean-Baptiste Grison \\ Docteur en géographie, laboratoire CERAMAC (Université de Clermont-Ferrand) \\ ATER à l'université de Nantes
}

\begin{abstract}
Résumé
Les communes françaises de moins de cinquante habitants représentent une part marginale du maillage administratif national. Cependant, l'étude de leur répartition et de leur organisation apportent des éléments intéressants de compréhension de certains aspects des espaces ruraux français. La régionalisation nationale de ces entités met en valeur une combinaison de facteurs, de la densité de population aux héritages de la Révolution Française. A plus grande échelle, on constate notamment que les grandes lignes de reliefs peuvent aussi intervenir dans ce découpage. Au-delà de ces constats, il ressort que la très petite commune est une clé d'entrée originale dans l'observation de la démographie des régions rurales, ou encore des logiques périurbaines. Elle ouvre aussi un autre regard sur la comparaison des maillages administratifs européens et met en lumière le poids de l'effet taille dans les analyses statistiques construites à l'échelle municipale. Enfin, les problématiques spécifiques aux communes de très petite taille enrichissent le débat autour de l'administration et de la politique territoriales. Les conséquences du morcellement communal sont finalement à double tranchant, entre la faiblesse du potentiel d'action inhérente au nombre réduit des habitants, et l'intérêt de la proximité de la population.
\end{abstract}

Mots clés :

France - Géographie administrative - développement rural - politique locale - dynamiques socio-spatiales - démographie. 
Longtemps délaissés par la géographie classique issue de l'école vidalienne, les découpages administratifs rencontrent un intérêt plus marqué, depuis quelques décennies, auprès des géographes contemporains. Cet engouement est lié, avant tout, au développement de nouvelles formes de gouvernance locale, qui posent des questions nouvelles de délimitations de périmètres, de recherche de cohérence territoriale, ou d'identités locales émergentes. Le grand mouvement de mise en place des pays et des intercommunalités fiscalisées a ainsi fait l'objet d'une littérature relativement fournie.

En revanche, l'ossature administrative traditionnelle, héritée de la Révolution Française, et qui persiste malgré l'avènement de ces nouvelles structures, est peu présente dans la production scientifique contemporaine de notre discipline. Pourtant, la commune, premier échelon du découpage territorial français, est très utilisée en tant que support statistique, sans que son contenu et sa signification soient souvent discutés. En réalité, l'institution municipale, largement débattue dans le cadre des réformes (ou tentatives de réformes) actuelles de l'organisation territoriale nationale, présente un intérêt géographique évident. Pour aborder cette question, nous avons choisi de nous concentrer sur la seule signification des communes françaises les moins peuplées, celles qui comptaient moins de cinquante habitants lors de l'estimation de 2006. L'objectif n'est pas, ici, de mettre en évidence un sens précis de ce seuil démographique, mais de montrer, d'une part, l'originalité géographique de l'étude d'une catégorie municipale, et d'autre part, la contribution potentielle de ce type d'étude à des réflexions plus générales, en géographie ou dans d'autres disciplines, à propos des espaces ruraux et de l'aménagement du territoire.

\section{Analyse géographique d'un objet marginal}

Les communes de moins de cinquante habitants représentent un objet marginal à plus d'un titre. Non seulement, on en compte moins d'un millier (955 d'après l'estimation 2006), soit moins de $3 \%$ des municipalités françaises. Mais en outre, elles ne totalisent qu'une part infime de la population nationale (environ 33000 habitants concernés), et par leur faiblesse démographique, se positionnent nécessairement en situation de marge au sein de leurs territoires d'appartenance. Mais en dehors de ces observations générales, le géographe doit aussi s'intéresser à l'originalité tangible de la répartition régionale et sous-régionale des entités concernées, qui fait appel à des croisements de facteurs spatiaux méritant d'être approfondis. Au total, la superficie qu'elles occupent $\left(7600 \mathrm{~km}^{2}\right.$, soit l'équivalent d'un gros département français) leur donne d'ailleurs un peu plus de poids.

A. Dispersion nationale, singularités régionales : où sont les très petites communes ?

1. Des îlots dans le territoire national

Dans un premier temps, la carte de répartition par points des communes françaises de moins de cinquante habitants (Figure 1) présente un certain intérêt. Les différents degrés de fréquence de ces entités dessinent en effet une géographie singulière, mettant en valeur des clivages peu habituels dans les analyses connues de la France rurale.

$\mathrm{Au}$ niveau national, deux grandes régions de forte concentration de très petites municipalités se distinguent en priorité : le midi pyrénéen d'une part, le Nord-Est d'autre part. Deux autres ensembles de fréquences majeures, mais d'étendues moindres doivent encore être retenus : les Préalpes drômoises et la Châtaigneraie corse. A l'inverse, les régions d'absence 
de très petites communes reviennent d'abord à l'ouest du pays, à commencer par la Bretagne, qui n'a recensé aucune entité administrative en dessous de la cinquantaine de résidents.

Figure 1 - Les communes de moins de cinquante habitants en France

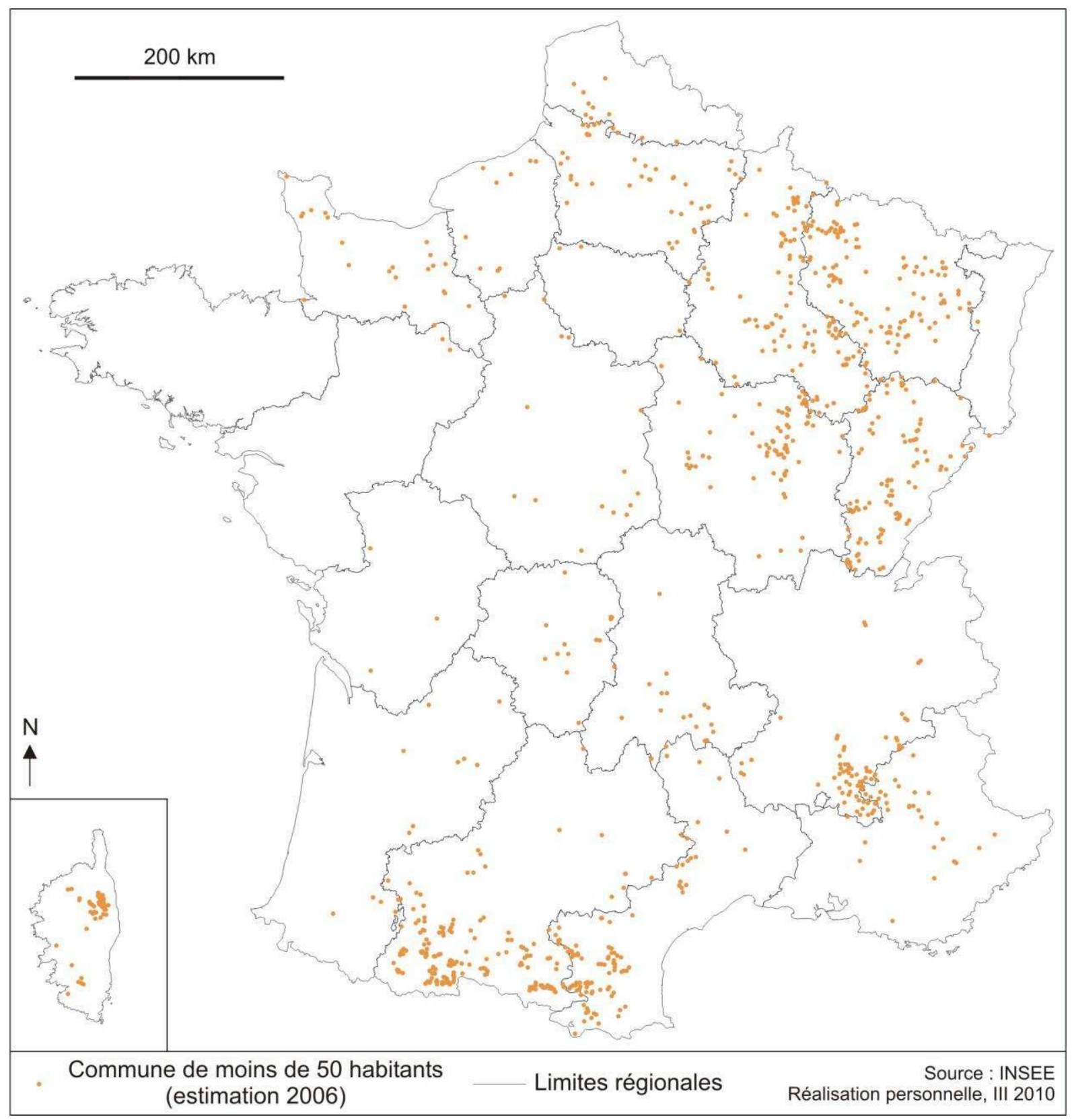

Cette première approche spatiale de la répartition des très petites communes françaises pose déjà des questions élémentaires : comment les Pyrénées et les plateaux du Nord-Est peuvent-ils connaître une problématique similaire dans ce domaine, alors qu'il s'agit à l'évidence d'espaces aux caractéristiques par ailleurs très différentes ? Pourquoi des espaces de très faibles densités de population tels que le Massif central ne présentent qu'une fréquence modérée de municipalités de moins de cinquante habitants ? D'emblée, la géographie des très petites communes nous conduit à revisiter quelques grandes problématiques propres à l'étude des espaces ruraux français. 
2. Des situations géographiquement singulières

Au-delà de l'observation de la répartition nationale des localités les moins peuplées, leur insertion dans les trames administratives régionales ou locales permet d'affiner davantage les réflexions. A première vue, on pourrait penser que la répartition des plus petites entités renvoie aux inégalités de la trame administrative de base : y compris dans les régions où elles sont les plus présentes, elles ne forment jamais un semis régulier, mais s'agrègent en ensembles plus ou moins compacts. A partir de quelques exemples, il semble ainsi judicieux d'approfondir les caractéristiques de cette distribution.

Figure 2 - Communes de moins de cinquante habitants sur les plateaux de Bourgogne

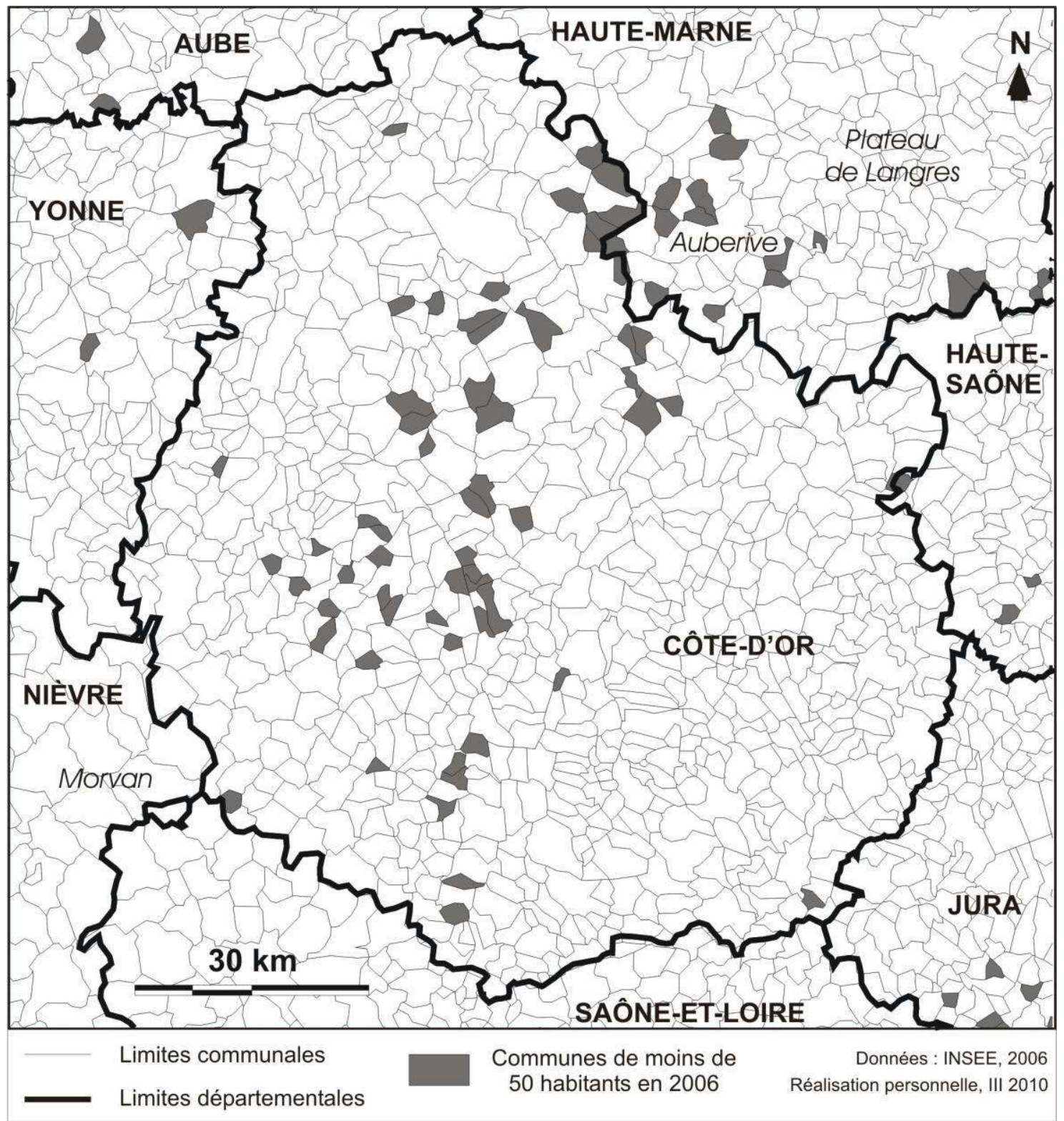

$\mathrm{Au}$ sein des fortes fréquences de très petites entités du Nord-Est de la France, les plateaux de Bourgogne, dans le département de la Côte-d'Or, tiennent une bonne place. En effet, au sein de ce département très pourvu en communes de moins de cinquante habitants, il 
est évident que celles-ci ne s'y localisent pas de manière homogène (Figure 2) : leur présence est presque réservée à la partie occidentale, et plus exactement, une bande allant des confins septentrionaux du Morvan au plateau de Langres. Si la région est globalement caractérisée par un morcellement sensible du tissu administratif, la relation à plus grande échelle n'est pas vérifiée, puisque les surfaces municipales sont plus réduites, en moyenne, à l'est de la Côte d'Or, dans la plaine. En revanche, à l'échelle du plateau, les localités les moins peuplées contrôlent des territoires généralement plus réduits que la moyenne. On peut en conclure que, même si le morcellement administratif est un facteur majeur de la présence de villages de moins de cinquante habitants dans les territoires ruraux, ceux-ci relèvent aussi de conditions environnementales et territoriales plus complexes.

\section{Figure 3 - Communes de moins de cinquante habitants en Lorraine}

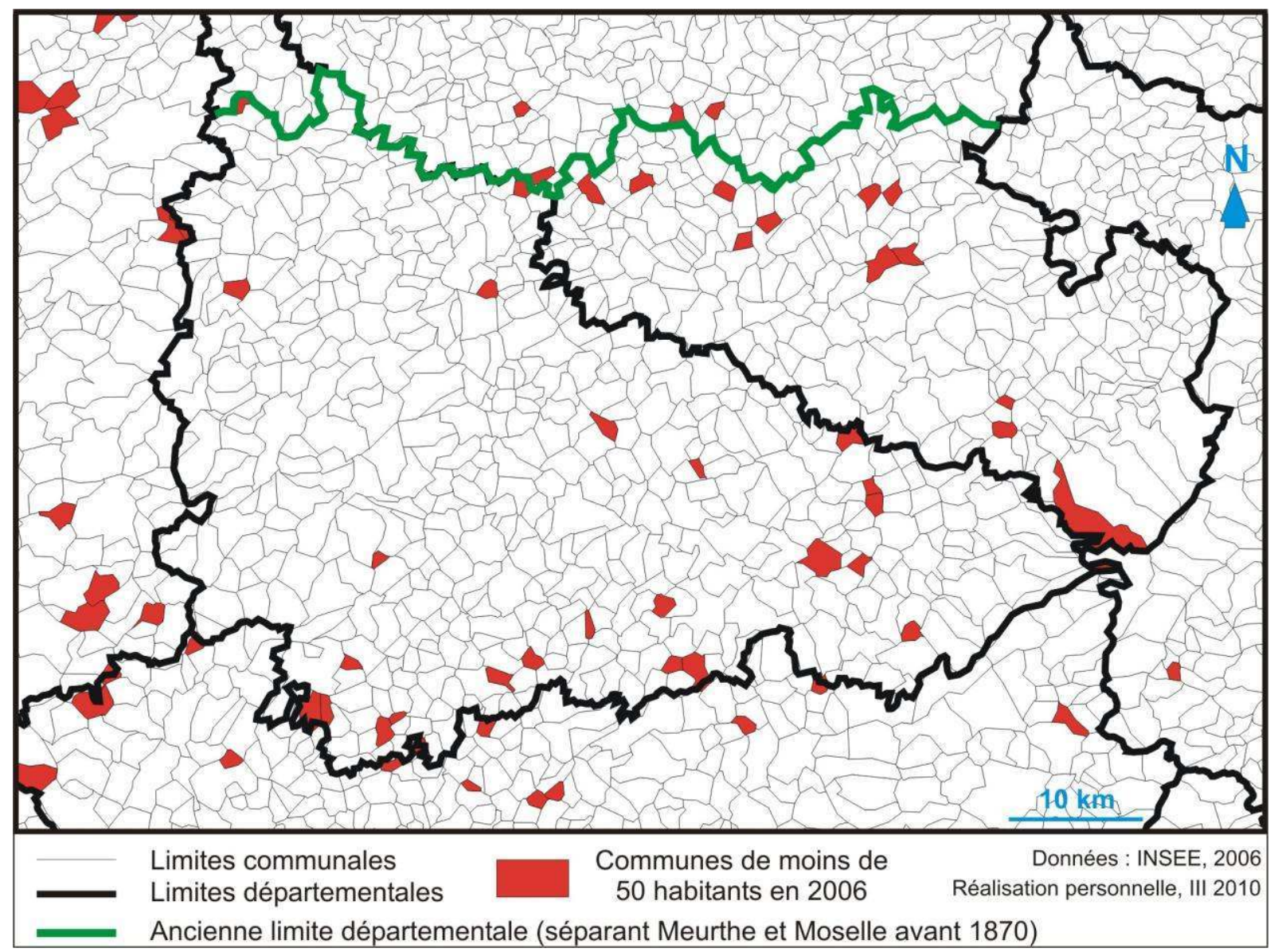

Plus au Nord, les municipalités étudiées sont moins présentes au cœur de la Lorraine, mais leur répartition offre quelques aspects intéressants (Figure 3). Comme dans le cas précédent, elles apparaissent parmi les cellules administratives les moins étendues, cependant, elles se situent cette fois-ci dans les espaces où la trame communale est la plus morcelée. En outre, on reconnaît dans leur distribution un effet de confins manifeste : les très petites communes sont sensiblement plus courantes à proximité de la limite sud du département de la Meurthe-et-Moselle; quant à la localisation des plus septentrionales, elle laisse aisément deviner le tracé approximatif de l'ancienne limite de la Meurthe et de la Moselle, avant le partage consécutif à la défaite française de 1870. Ainsi, l'état de marginalisation des entités de moins de cinquante habitants est bien souligné, et appuie au passage une certaine cohérence des périmètres départementaux constitués lors de la Révolution Française ; cet aspect sera 
examiné ultérieurement. D'autres régions présentent une organisation similaire, comme la Picardie.

En Haute-Corse, le massif de la Castagniccia (Châtaigneraie corse) comprend un des ensembles les plus concentrés de très petites communes jointives (Figure 4). L'observation de la trame administrative montre que dans ce cas, l'émiettement des contours municipaux joue un rôle majeur dans cette situation. En effet, cette région au relief très découpé a été peuplée sous la forme de petits villages dont les finages sont restés bien distincts les uns des autres. En périphérie de ce massif, les entités étudiées sont moins présentes, et leur liaison avec le degré de morcellement des limites territoriales apparaît moins systématique. On repèrera néanmoins le cas d'une localité de superficie très réduite dans l'arrière-pays de l'Île-Rousse, encadrée par des villages dont le territoire est plus étendu.

Figure 4 - Communes de moins de cinquante habitants au nord de la Corse

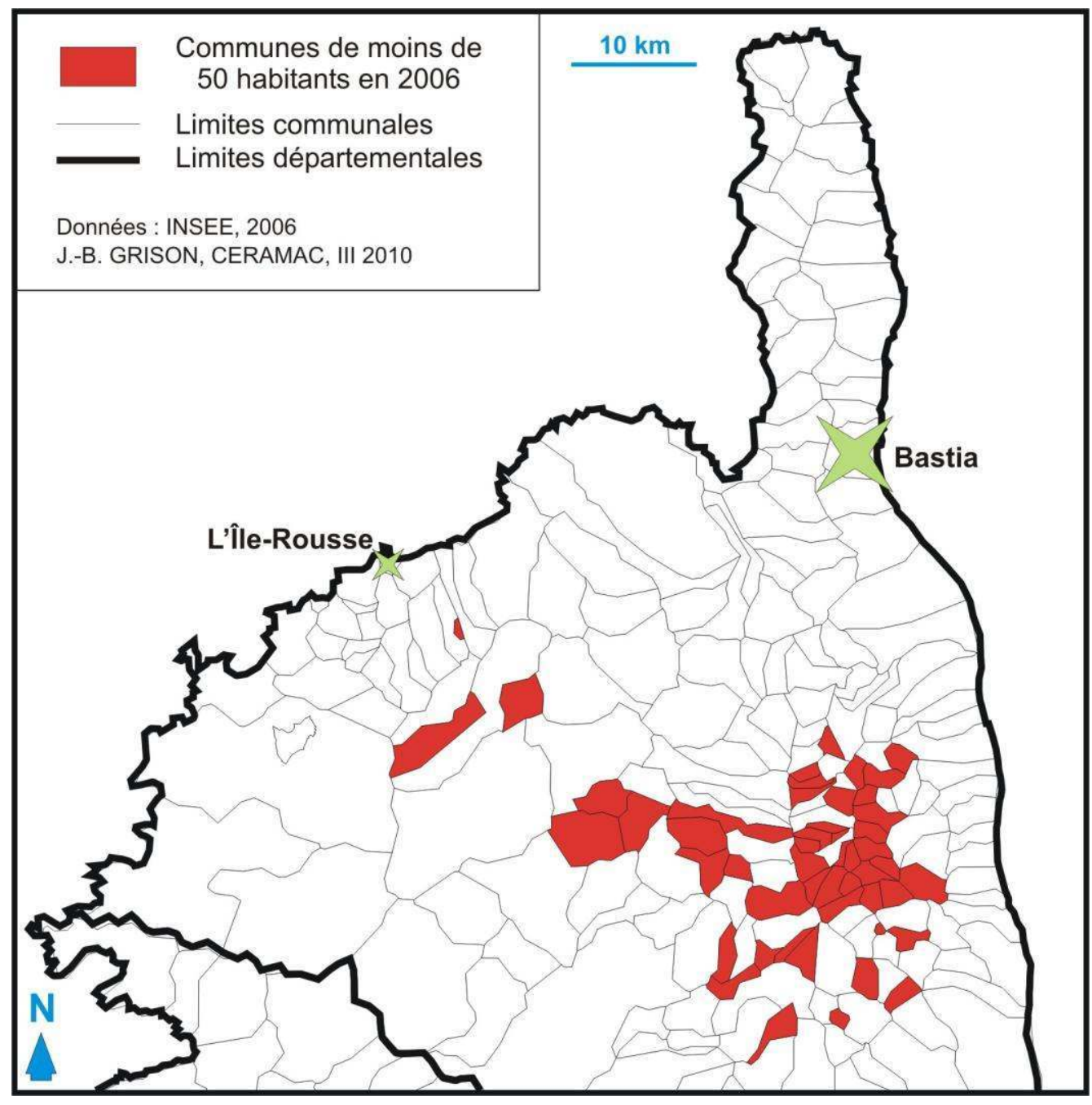

A l'échelle nationale comme au niveau des régions, la répartition des très petites communes ne se passe pas de commentaires. Les irrégularités de leur distribution mettent en valeur quelques régions de fortes fréquences aux caractéristiques pourtant très différentes, qui laissent supposer que des facteurs variés en sont à l'origine. Au sein des régions concernées, il 
s'avère que les facteurs physiques, mais aussi la dialectique centre périphéries interviennent dans la localisation des municipalités les moins peuplées. Inversement, on peut considérer ces dernières comme un indicateur soulignant des caractères locaux particuliers.

\section{B. Logiques de répartition : quelques essais d'interprétation}

L'originalité de la répartition des très petites communes françaises mérite que l'on s'attarde quelque peu sur les facteurs potentiels qui la provoquent. En effet, la municipalité de moins de cinquante habitants fait appel, a priori, à plusieurs phénomènes. Le premier est le rapport étroit qu'entretiennent morcellement administratif et densités de population: les exemples locaux choisis précédemment l'ont montré. D'autres facteurs interviennent ensuite, comme la dispersion de l'habitat et le degré de métropolisation, les dynamiques démographiques des territoires, mais aussi les grandes formes de relief.

La trame communale française est avant tout un produit de la Révolution Française, période à laquelle elle a été constituée, et depuis laquelle elle n'a été modifiée que marginalement. Cette trame, si elle a abouti globalement à un morcellement considérable, et dont la survivance est unique en Europe à l'échelle d'un pays de cette envergure, n'en présente pas moins des irrégularités régionales significatives. Tout d'abord, la trame des paroisses de l'Ancien Régime a formé, en général, la base du maillage révolutionnaire. Or, les études historiques ont bien montré que le tissu paroissial, dès cette période, n'était pas homogène : très dense dans les régions où le développement de l'Église était le plus ancien, il était un peu plus épars dans d'autres, notamment en Bretagne où son installation n'a pas été concomitante, mais postérieure au défrichement des terres et au développement du système d'habitat. Ensuite, dans certains départements, et tout particulièrement dans un large quart Nord-Est, de nombreuses localités qui n'étaient pas des paroisses ont néanmoins été converties en municipalités. Ainsi, en Franche-Comté, une grande partie des actuelles communes de moins de cinquante habitants ne compte pas d'église, alors que ce monument est ailleurs un repère patrimonial marquant dans les identités locales.

Les très petites entités sont nécessairement le reflet, au moins au niveau local, de faibles densités de population. Il est cependant évident que la distribution de ces entités sur le territoire national est très éloignée de la carte générale de répartition du peuplement: le Massif central compte relativement peu de localités concernées, alors qu'il se situe au cœur de ce qui est souvent appelé la «diagonale du vide », le long de laquelle s'alignent des espaces très faiblement peuplés. Par ailleurs, trois départements seulement font partie, à la fois, des dix les moins densément peuplés, et des dix comptant le plus de communes de moins de cinquante habitants.

La combinaison de la densité de population et de celle du maillage communal permet d'expliquer en partie la fréquence des très petites municipalités. Par exemple, le Nord-Est et les Pyrénées combinent faibles densités de population et taille réduite des cellules administratives, tandis que la Bretagne compte des communes vastes et des densités de population plus élevées. Le Massif central présente un peuplement faible, mais les surfaces municipales relativement étendues limitent sensiblement le nombre d'entités de moins de cinquante habitants. C'est ainsi que la Lozère, l'Aveyron et le Lot totalisent, à eux trois, autant de très petites localités que l'aire urbaine de Paris, où le morcellement administratif compense, dans ce domaine, des densités de population sensiblement plus importantes. En 
somme, les fréquences significatives de très petites entités supposent la combinaison d'une densité de population faible et d'un fort émiettement administratif.

Une fois cette règle générale reconnue, d'autres éléments peuvent être mis en perspective avec la répartition des très petites communes. Par exemple, l'alternance, dans les territoires ruraux français, de la dispersion et de la concentration de l'habitat, pourrait, a priori, rappeler la régionalisation observée des entités étudiées. En effet, à première vue, les régions qui comptent des municipalités vastes et de moins de cinquante habitants (cas de figure assez rare), correspondent principalement à des régions d'habitat dispersé : l'ensemble du centre-ouest du pays, de la Bretagne au Massif central, répond à ce critère. On comprend aisément que l'habitat dispersé favorise des structures administratives de base plus étendues, en raison d'une fréquence moindre des places de centralité locale (bourgs, villages). Pourtant, cette relation ne se vérifie pas partout : des communes de moins de cinquante habitants sont présentes en nombre significatif dans certaines zones de Normandie ou du piémont pyrénéen, où l'habitat est pourtant très dispersé : dans ces conditions, les localités étudiées sont souvent des localités sans village, simples regroupements de fermes et hameaux restés indépendants pour des raisons diverses. Parfois aussi, elles représentent des exceptions d'habitat groupé dans un environnement où la dispersion est de mise.

Au-delà de la simple appréhension des densités de population, les dynamiques démographiques influent sur l'évolution de la localisation des très petites communes : leurs régions de présence historique et structurelle, liée aux causes précédemment évoquées, ne sont plus tout à fait les mêmes que les grands ensembles actuels de leur concentration. D'une part, un exode rural considérable a touché, de la fin du dix-neuvième siècle au dernier quart du vingtième, une grande partie des campagnes françaises, et son ampleur inégale, comme son décalage dans le temps d'une région à l'autre a modifié les rythmes d'apparition de nouvelles localités à moins de cinquante habitants. D'autre part, les phénomènes d'extension des aires urbaines (qui sont aujourd'hui les principaux espaces de la croissance démographique rurale), ont permis, depuis les années 1960 pour certains territoires, la diminution du nombre de municipalités concernées par notre seuil d'étude.

Par exemple, la concentration de très petites communes en Castagniccia, observée précédemment, apparaît comme un marqueur tangible d'un héritage démographique singulier : la « région aux mille villages » porte dans son découpage administratif les traces d'un passé de région rurale parmi les plus densément peuplées d'Europe (au XIX ${ }^{\mathrm{e}}$ siècle, plus de 50 habitants par kilomètre carré en moyenne, et plus d'une centaine dans certaines communes), avant un déclin colossal qui aboutit à la situation originale actuelle.

En somme, dès sa création, l'institution communale comptait des unités très peu peuplées, comme en témoignent d'ailleurs certains rapports de l'époque. Mais ces microstructures n'étaient alors présentes que dans quelques régions, auxquelles l'émiettement du maillage était favorable. Ces régions ne correspondaient pas réellement à la carte des densités de population. Le dépeuplement de nombreux territoires isolés, puis la métropolisation d'autres ensembles mieux situés, ont amplifié, puis modifié la trame des municipalités de moins de cinquante habitants, qui se rapproche davantage, aujourd'hui, de l'espace « rural profond », tout en conservant des irrégularités notoires liées à leur histoire.

\section{Les très petites communes : quels apports disciplinaires ?}

Peu abordé par les géographes jusqu'à présent, le thème des très petites communes (ou, plus généralement, de la taille des municipalités) représente à nos yeux un élément 
intéressant dans l'étude géographique des territoires. Concernant les dynamiques des espaces ruraux, on peut émettre l'hypothèse que l'évolution de la fréquence de ces municipalités peut être un indicateur à considérer, de même que la prise en compte de leurs tendances dominantes. La question des localités les moins peuplées éveille aussi le problème des inégalités de taille des cellules d'une trame qui sert de base à de nombreux travaux, et de l'influence que cette hétérogénéité peut avoir sur les résultats observés. Nos investigations ont en outre permis de mettre en évidence la place originale des plus petites entités rurales dans les processus de recompositions territoriales, et les nouveaux modes de gouvernance locale qui y sont associés. Enfin, les effectifs réduits des communautés locales concernées sont sources d'approches particulières en géographie sociale.

\section{A. Un indicateur pour l'étude des espaces ruraux}

Les remarques à propos de l'explication de la répartition des très petites communes françaises mettent déjà en lumière une relation, complexe mais réelle, entre la fréquence de ces localités, son évolution, et certaines caractéristiques et dynamiques des espaces ruraux. On peut considérer que, sous des modalités variables suivant les régions, les municipalités de moins de cinquante habitants contribuent à l'identité des territoires. On peut ainsi retenir deux thématiques majeures que notre objet d'étude permet de revisiter d'un regard original.

\section{Les empreintes de la crise et de la renaissance rurales}

A juste titre, l'ampleur du phénomène des communes de moins de cinquante habitants à la fin du vingtième siècle peut être considérée comme une conséquence évidente de la grande vague d'exode qui a frappé les espaces ruraux dans les décennies précédentes. Cependant, la réduction à l'extrême des effectifs démographiques a pris une ampleur variable, et donné une consistance particulière à certains espaces. Ainsi, dans les Alpes du Sud, la Castagniccia, l'est des Pyrénées, des vallées entières se sont vidées en donnant un sentiment d'abandon, même si les cas de dépopulation totale sont restés rares (un peu plus fréquents néanmoins en haute Provence). Dans ces moyennes ou hautes montagnes, la présence en nombre de très petites localités prend aujourd'hui la valeur patrimoniale de l'héritage d'un peuplement très dense, où les équilibres anciens ont été totalement rompus. Le morcellement administratif actuel témoigne ainsi de la surpopulation passée, qui avait permis, au début du dix-neuvième siècle, de donner un statut municipal à tant de villages.

Dans le Massif central, la trame administrative plus lâche, liée, nous l'avons vu, à une dispersion plus sensible de l'habitat, mais aussi à une tradition plus marquée qu'ailleurs des sections de commune, est longtemps restée peu favorable à l'existence de municipalités de moins de cinquante habitants (Couturier, 2000). Celles-ci sont apparues, en nombre modéré, bien plus récemment que dans les montagnes précédemment citées. Elles marquent ainsi une poche de persistance du déclin démographique que constitue ce centre-sud de la France : entre 1999 et 2006, plusieurs départements du Massif central appartiennent à la minorité de ceux où le nombre de communes de moins de cinquante habitants s'est accru.

Dans le Nord-Est, les plateaux orientaux du Bassin parisien connaissent aussi une érosion démographique persistante, mais le nombre de communes très peu peuplées est stable, voire en diminution au cours des deux dernières décennies. Par ailleurs, la perte globale de population est restée moindre que dans les montagnes du sud de la France : le surpeuplement passé n'était pas aussi important. Cette observation nous conduit à émettre l'hypothèse suivante : les espaces ruraux de ces régions conservent un socle d'occupation humaine du 
territoire qui, une fois descendu à un certain seuil, se stabilise. Le maintien d'une agriculture qui, si elle emploie de moins en moins d'actifs, continue à tenir l'espace et reste globalement compétitive, expliquerait alors cette stabilisation des entités les moins peuplées; la persistance du déclin démographique serait le fait des localités plus importantes, à commencer par les bourgs et petites villes, dont la situation économique est souvent difficile.

Ces trois exemples montrent que l'étude des très petites communes fait ressortir des nuances dans le déclin démographique des campagnes françaises, en les plaçant sous un éclairage original. Sans être en mesure d'affirmer que le seuil de cinquante résidents ait une signification en tant que tel (des études régionales pourraient justifier des seuils variables), nous pouvons déjà souligner que l'analyse des localités par catégorie présente un intérêt dans la compréhension des dynamiques territoriales.

\section{Territoires éponges ou territoires remparts face à l'urbanisation}

Si les très petites communes reflètent d'abord l'idée de déclin démographique et d'isolement géographique, leur étude contribue aussi à mettre en relief certaines tendances relevant de dynamiques métropolitaines. En premier lieu, la diminution du nombre de municipalités de moins de cinquante habitants traduit souvent, à travers le regain de population des villages, une extension des phénomènes périurbains jusqu'à ces petites localités. Si celles-ci sont souvent localisées, à l'origine, en situation de marge, leur regain marque alors une progression d'un front périurbain, qui précède, dans certains cas, leur intégration avérée dans le périmètre d'une aire urbaine telle que l'INSEE l'a définie. La localisation des concentrations d'anciennes communes de moins de cinquante habitants est intéressante à ce sujet: on se rend compte que les alentours de Besançon, mais aussi les confins du Bassin parisien et de la Normandie, la bordure méridionale des Alpes, sont largement concernés. Ces entités, qui restent relativement peu peuplées, traduisent un développement, souvent léger mais bien visible à cette échelle, de dynamiques résidentielles nouvelles, auxquelles des grandes métropoles, pourtant éloignées de plusieurs dizaines de kilomètres, ne sont pas étrangères.

Dans d'autres situations, la présence de très petites municipalités à proximité d'agglomérations importantes, souvent isolées dans leur cas, et entourées de villages plus développés, soulève d'autres questions. Des enquêtes et observations personnelles ont en effet montré l'existence d'une résistance municipale, ponctuelle mais répétée à de multiples reprises, face à la croissance périurbaine (Grison, 2008, 2009). Dans de tels cas, la persistance d'une localité de moins de cinquante habitants, héritée à la fois d'une marginalisation passée et d'une configuration locale (et notamment foncière) originale, témoigne de la volonté d'une communauté locale de ne pas céder à une pression urbaine pourtant significative. Des logiques de blocage foncier et le développement de dispositifs juridiques de protection de l'espace appuient généralement une telle situation.

Finalement, au travers de ces deux thématiques que sont la succession de la crise et de la renaissance rurales, d'une part, et les dynamiques métropolitaines, d'autre part, la dialectique entre centralité et marginalisation est bien présente. On peut considérer, d'une certaine manière, que les communes de moins de cinquante habitants permettent d'aborder plus finement les enjeux et conséquences de la hiérarchie des lieux au sein des territoires. 
B. Comparer des territoires : questionnement méthodologique autour de l'effet taille

L'exploration du thème des très petites communes est aussi l'occasion de mises au point méthodologiques relatives à l'analyse des espaces ruraux. S'il est peu courant d'étudier, en géographie, les dynamiques territoriales en prenant en compte l'influence des tranches démographiques des municipalités, il est en revanche assez habituel de commenter des données statistiques diffusées à l'échelle communale. Or, l'observation des variations de fréquence des unités de moins de cinquante habitants au sein des régions françaises nous a permis de mettre au jour plusieurs biais liés à un «effet taille» des mailles considérées, dont la première conséquence se situe dans les difficultés de comparaison de plusieurs territoires à configurations différentes.

\section{Des comparaisons internationales : exemples européens}

La trame administrative française est l'une des plus morcelées d'Europe. Les équivalents de la commune dans les états voisins sont généralement plus proches, d'après les moyennes générales, du niveau cantonal (ou intercommunal). La nomenclature européenne des unités administratives locales (LAU) en tient compte partiellement, en reconnaissant deux niveaux (LAU 1 et LAU 2) pour les comparaisons. Seulement, l'observation attentive des localités les moins peuplées dans les pays de l'Union Européenne montre que les cellules de moins de cinquante habitants peuvent être présentes de manière significative dans certaines régions de pays pour lesquels les moyennes nationales sont plus proches du niveau LAU 1 (cantons français) que de la classe LAU 2 (communes françaises). Les exemples de l'Espagne et de l'Allemagne sont assez frappants à ce titre.

En effet, 1'Espagne, avec seulement 8000 municipios (soit quatre fois moins qu'en France, pour une superficie inférieure de $10 \%$, et une population inférieure de $35 \%$ environ), présente des moyennes bien supérieures aux nôtres. Pourtant, ce pays est celui en Europe où la proportion d'entités de moins de cinquante habitants est la plus importante : le déséquilibre très important entre les métropoles où se concentrent une grande part de la population, et des campagnes aux densités extrêmement faibles, explique cette situation.

En Allemagne, avec environ 12000 Gemeinden pour près de 80 millions d'habitants, la répartition démographique moyenne est également plus proche de celle des cantons français, laissant supposer une certaine puissance de l'institution locale. Cette observation trop générale tend en réalité à faire oublier les disparités profondes entre les Länder : toute proche de la frontière alsacienne, la Rhénanie-Palatinat compte plusieurs dizaines d'entités de moins de cinquante habitants, et à elle seule près de $20 \%$ des municipalités allemandes... A l'inverse, le Luxembourg et l'Autriche, dont la trame morcelée rappelle celle de la France, présentent une plus grande homogénéité et ne recensent finalement presque aucune entité (aucune au Luxembourg, cinq en Autriche) en dessous de notre seuil d'étude.

Dans ces conditions, des problèmes délicats de comparaisons se posent. Si les moyennes nationales peuvent amener des conclusions faciles, elles tiennent difficilement lorsque l'on s'attaque aux irrégularités régionales. Faut-il comparer les municipalités allemandes et espagnoles aux communes ou aux cantons français ? Tout dépend, finalement, de la région que l'on cherche à mettre en valeur. 
2. Des comparaisons au sein de l'espace français

Les problèmes de comparaison peuvent aussi se poser au sein même des territoires ruraux français. Nous avons vu, en effet, que la structure de l'habitat et celle de la trame communale peuvent différer sensiblement d'un territoire à l'autre, y compris à densité égale de population. L'analyse du niveau de desserte des équipements et services publics est un exemple très instructif, car fortement révélateur des biais qui peuvent s'introduire dans des analyses trop rapides. Une petite comparaison, à titre d'exemple, de quelques localités du Ségala aveyronnais avec quelques villages de la vallée de l'Ariège paraît judicieuse (Figure 5).

Figure 5 - Comparaison des trames administratives en Ariège et Aveyron

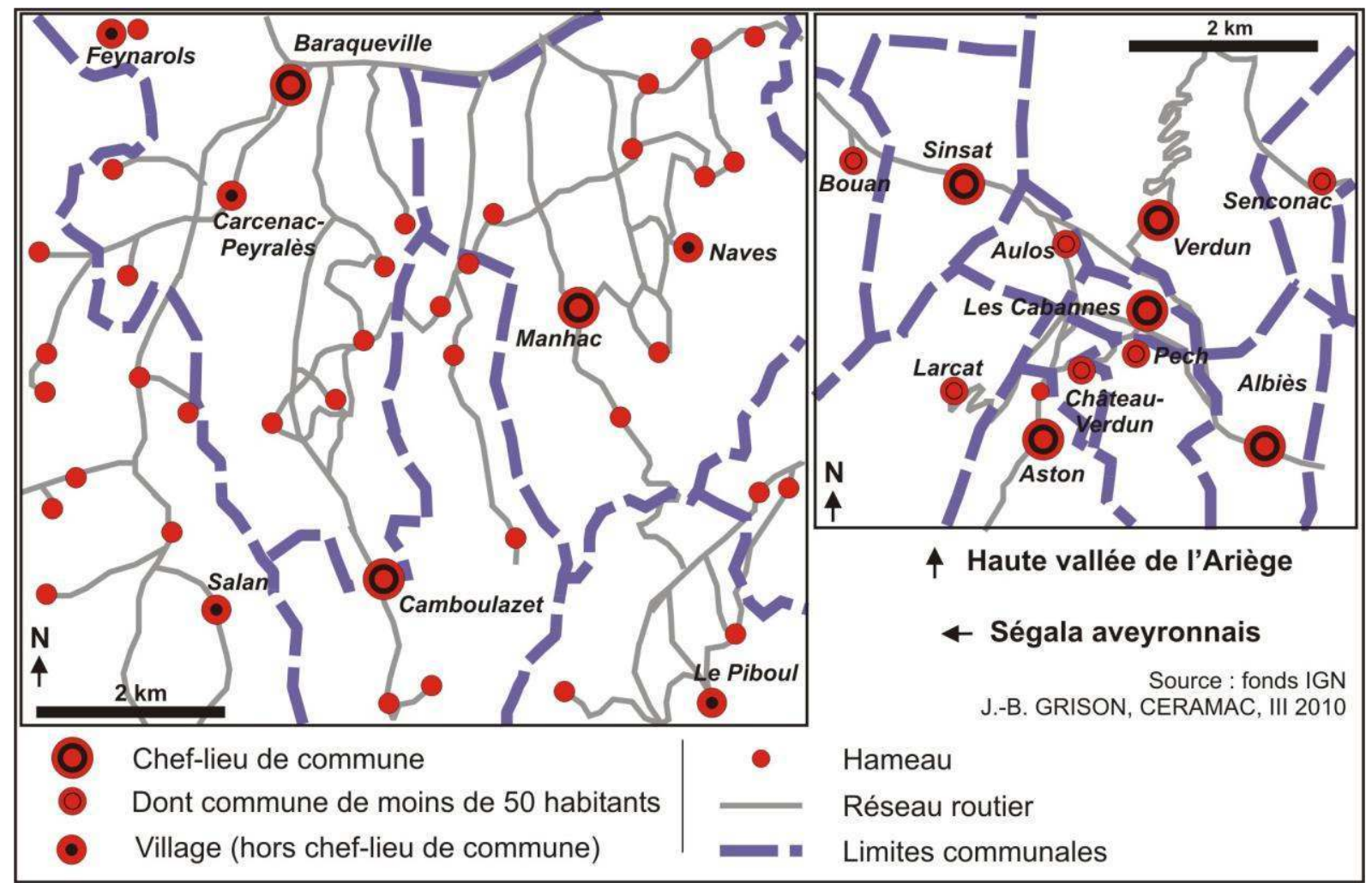

Tout d'abord, en rapportant le nombre d'habitants à la superficie des communes représentées, les densités de population sont plus faibles en Ariège. En réalité, l'observation de la carte montre bien que la proximité des espaces bâtis, ainsi que la qualité de leur accessibilité, favorisent plutôt ce dernier. Evidemment, nous retiendrons, concernant la trame municipale, que le morcellement est sensiblement plus important en Ariège, où chaque village bénéficie de son autonomie administrative (plus de la moitié comptant moins de cinquante habitants), tandis qu'en Aveyron de nombreuses localités (y compris d'anciennes paroisses de l'Ancien Régime) sont rattachées à un bourg plus important et distant de quelques kilomètres.

Il est bien entendu possible de discuter l'intérêt de l'autonomie de tous les villages ariégeois, ou le manque d'individualité des hameaux aveyronnais, dans le cadre d'une telle comparaison. Cependant, nous souhaitons ici attirer l'attention sur un point précis : quelle est la portée de la signification réelle du taux d'équipement des communes ariégeoises ou aveyronnaises de tel ou tel service? A titre d'exemple, l'équipement scolaire des deux zones 
considérées doit être analysé avec une certaine prudence. D'après l'inventaire communal, dans le Ségala, trois communes sur les cinq représentées comptent une école ; dans l'Ariège, seules deux localités sont équipées, en se partageant un unique regroupement scolaire : pour les onze entités représentées, il n'y a donc qu'une classe par niveau.

Une observation trop hâtive de cette répartition, au regard du pourcentage de communes équipées (plus de la moitié dans l'Aveyron, à peine $10 \%$ en Ariège), ferait état d'un important écart d'équipement entre les deux départements. En réalité, la répartition de la population au sein de l'espace en question invite à tempérer considérablement ce constat : en effet, dans quelle mesure les habitants de Pech, Château-Verdun ou Aulos, municipalités ariégeoises, seraient-il moins bien desservis que ceux de Feynarols, Salan ou Le Piboul, villages de l'Aveyron dépendants d'entités équipées, mais éloignés de plusieurs kilomètres du chef-lieu ? Finalement, la dispersion de l'habitat en Aveyron, si elle n'est pas prise en compte, risque de biaiser la compréhension de la réalité locale. A l'inverse, la grande proximité des chefs-lieux de communes ariégeois, malgré des densités de population bien plus faibles (traduisant l'existence de larges espaces vides), permet de considérer qu'une absence d'équipement de l'un d'eux n'empêche pas une bonne accessibilité à la desserte, même si ponctuellement, certains villages restent très isolés, comme ici celui de Senconac.

Cet exemple prouve que l'on ne peut pas considérer la trame communale française comme un critère de comparaison efficace de plusieurs territoires, en cas de disparités trop significatives. Dans certaines mesures liées à l'aménagement du territoire, des directives trop homogènes peuvent avoir des conséquences déséquilibrées localement: nous avons ainsi observé, dans le cadre de politiques régionales de résorption des zones blanches en matière d'accessibilité aux réseaux des NTIC (téléphonie mobile, haut débit Internet), que le fait de vouloir équiper tous les chefs-lieux de commune aboutit à desservir une part très variable de la population, et dans ce cas, les trames les plus morcelées s'en sortent mieux.

Dans ces conditions, le faible contenu des cellules de moins de cinquante habitants ne doit pas être observé comme la traduction automatique d'une situation d'isolement et de sous équipement territorial systématique. La marginalisation peut ainsi être relativisée.

\section{Recompositions territoriales et originalités politiques des très petites communes}

Si les relations entre très petites localités et dynamiques rurales restent étroites, si la prise en compte de la taille des municipalités aboutit à des problématiques d'aménagement différenciées, on peut alors supposer que les communes de moins de cinquante habitants s'insèrent de manière originale dans le «mille-feuille» des collectivités territoriales, et méritent une attention particulière au regard des multiples études sur les recompositions contemporaines de la gouvernance locale.

\section{Les très petites communes et la carte de l'intercommunalité}

Le développement des communautés de communes et d'agglomération représente à l'évidence une évolution majeure des deux dernières décennies, dans la vie des collectivités municipales. Cette évolution est supposée résoudre les difficultés rencontrées par les petites communes à assumer les compétences dont elles ont la charge, en les mutualisant. Cependant, la place, au sein de ces nouveaux périmètres, des localités les plus restreintes, soulève des questions pertinentes relatives à leur intégration, et la qualité de la cohésion des nouvelles structures. 
Les résultats d'une enquête diffusée en $2007^{1}$ auprès des maires des communes étudiées ont montré que la bonne intégration des communes de moins de cinquante habitants dans les structures intercommunales à fiscalité propre dépend en bonne partie de la nature et de la qualité des relations avec l'exécutif du chef-lieu. Assez naturellement, ces relations s'inscrivent dans un dialogue centre-périphérie, dont l'équilibre n'est pas toujours un exercice facile. Tout d'abord, les intérêts et attentes des populations du bourg-centre ou de la petite ville ne sont pas systématiquement semblables. Il arrive que des concurrences de projets soient déplorées, par exemple entre les villages, pour l'entretien de la voirie ou des bâtiments publics, et le bourg-centre, qui émet des demandes pour améliorer ses équipements culturels. D'autres divergences apparaissent à propos de la nature des compétences attribuées : les plus petites entités ayant plus de difficultés à assurer, par exemple, l'entretien des réseaux d'adduction d'eau et d'assainissement, peuvent déplorer que les localités dominantes privilégient des attributions telles que le développement culturel, voire la mutualisation des documents d'urbanisme, qui peut aboutir à un sentiment de perte de contrôle de la part des «petits » élus, et une tendance au renforcement permanent de la centralité du chef-lieu. Evidemment, le nombre de municipalités adhérentes joue, lui aussi, un rôle sur l'intensité de l'intégration intercommunale: un nombre important de municipalités représente ainsi un handicap à la bonne cohésion du conseil communautaire (Grison, 2009).

L'observation des très petites communes et de leur insertion intercommunale peut aussi permettre un apport géographique sur les thématiques, largement pluridisciplinaires, de l'aménagement du territoire et du développement local. Tout d'abord, les cas éventuels, évoqués précédemment, de conflit d'intérêts entre la localité périphérique et l'intercommunalité pose la question de l'échelle spatiale de l'intérêt général. D'une certaine manière, le morcellement administratif local déplace cette notion à très grande échelle, en la ramenant au niveau d'une localité, par l'expression de ses seuls citoyens. Le paradoxe survient lorsque le territoire, plus large, dans lequel la très petite commune s'insère et au niveau duquel sont menées les principales opérations d'aménagement ayant trait à la vie quotidienne, prend des orientations différentes. On peut citer ce maire de la Beauce, très mal à l'aise devant la décision du chef-lieu de canton de construire une piscine intercommunale, à laquelle il devrait participer, tout en considérant qu'elle n'intéressera pas les habitants de son village, pour lesquels elle ne sera qu'à peine plus proche que celle existant déjà dans une ville voisine... Pourtant, un tel équipement n'aurait pu être envisagé, en zone rurale, sans la contribution d'un bassin de population dépassant largement un cadre communal unique.

En définitive, la très petite collectivité rurale présente la situation ambiguë d'une entité marginale et nécessairement incomplète en tant que territoire du quotidien, au regard des modes de vie contemporains, mais qui reflète fidèlement la trame historique des lieux habités, souvent porteurs d'une identité forte, et au sein desquels les communautés citoyennes locales trouvent souvent, aujourd'hui encore, une certaine cohérence. Ces observations nous montrent que les logiques politiques à l'œuvre dans des entités de moins de cinquante habitants sont très liées à des considérations sociologiques (ou socio-spatiales) spécifiques.

2. La politique municipale dans les très petites communes : une gestion originale du territoire

Les modalités officielles d'élection, puis de fonctionnement de l'exécutif municipal sont sensiblement les mêmes pour toutes les communes de moins de 3500 habitants.

\footnotetext{
${ }^{1}$ Dans le cadre d'un travail de doctorat, 547 questionnaires ont été renseignés par des maires de très petites communes (Grison, 2009).
} 
Cependant, la pratique est forcément différente : avec neuf conseillers à élire, des entités ne comptant que quelques dizaines d'électeurs (voire à peine dix dans les cas les plus extrêmes) ont un degré de représentation très supérieur à la moyenne. Ainsi, pour l'ensemble des municipalités françaises de moins de cinquante habitants, ce sont plus de $20 \%$ des électeurs qui sont ainsi élus. Dans ces conditions, il est courant de voir plusieurs membres d'une même famille, voire d'un même foyer, siéger au conseil : citons, comme cas extrême, l'exemple de Canteleux (Pas-de-Calais) où seuls trois patronymes différents apparaissent dans la liste de l'équipe politique.

Il résulte, de ces spécificités électorales, que les appartenances politiques des listes candidates ne sont pas tranchées au même titre que les idéologies nationales; en outre, il est souvent difficile d'opposer plusieurs listes complètes, quand des tensions existent. Dans les cas, minoritaires, où deux listes s'opposent, les argumentaires s'appuient sur des mésententes très concrètes. Par exemple, en Normandie, une commune enquêtée a connu un changement de majorité suite à une campagne centrée sur la préparation du plan d'urbanisme : l'équipe sortante souhaitait promouvoir quelques constructions nouvelles que les électeurs ont refusées au profit du maintien en l'état du périmètre bâti. Dans une autre commune, un nouvel équipement ayant provoqué un fort endettement a nourri la polémique. Parfois, l'opposition se cristallise entre anciens et nouveaux arrivants, résidents permanents et secondaires, habitants du chef-lieu et des écarts, ou font ressortir des luttes ancestrales entre vieilles familles. Quelquefois, nous avons pu sentir, en arrière-plan, des divergences de coloration politique, mais celles-ci restent inavouées. Et d'une manière générale, lorsqu'il existe deux listes (exceptionnellement trois), celles-ci restent incomplètes, et le débat peut se retrouver tel quel au sein de l'équipe élue. Enfin, dans la plupart des communes de moins de cinquante habitants, l'unique liste constituée comprend des candidats aux aspirations divergentes, et l'opposition émerge au fil du mandat.

En somme, la très petite localité autonome peut être considérée comme un modèle particulier de démocratie : d'un côté, le choix électoral est limité, mais de l'autre, l'exécutif au pouvoir reflète davantage la population que dans des unités plus importantes. Dans ces conditions, le débat semble être plus décisif au moment de la prise effective de décision qu'au moment du choix de l'exécutif.

Plus généralement, l'étude des communes de moins de cinquante habitants révèle des conditions originales dans l'action citoyenne et l'appropriation du territoire par les habitants. Ainsi, les projets municipaux font souvent appel à une part importante de travail bénévole des élus, notamment lorsqu'il s'agit de la valorisation des espaces collectifs. Globalement, la bonne connaissance mutuelle des habitants, et le cas échéant, un certain sentiment de faiblesse ou de marginalisation collective peut créditer un renforcement des solidarités, auxquelles le contour administratif donne une certaine reconnaissance.

\section{Conclusion}

Au-delà de l'originalité de leur répartition, prendre en considération les communes françaises de moins de cinquante habitants est l'occasion d'ouvrir des perspectives d'analyse dans des directions multiples : celle de la recherche, notamment, sur les espaces ruraux, celle davantage méthodologique et liée à l'aménagement à propos de la comparaison des territoires, une troisième enfin correspondant à des éléments de réflexion au sujet des réformes et des analyses politico-administratives appliquées à la strate municipale. 


\section{Bibliographie}

BÉTEILLE R., 1994, La crise rurale, Paris : Presses Universitaires de France (coll. Que saisje, $\left.\mathrm{n}^{\circ} 2914\right), 127 \mathrm{p}$.

CALMES R. et al, 1978, L'espace rural français, Paris : Masson, 192 p.

COUTURIER P., 2000, Sections et biens sectionaux dans le Massif central: héritage et aménagement de l'espace, Clermont-Ferrand: CERAMAC, Presses Universitaires BlaisePascal, 476 p.

DIRY J.-P., 1992, - «Commerces et services en moyenne montagne », in Des régions paysannes aux espaces fragiles, Clermont-Ferrand : CERAMAC, p. 247-258.

FRÉMONT A., 1997, France : géographie d'une société, Paris : Flammarion, troisième édition, $354 \mathrm{p}$.

GRISON, J.-B., 2008, « Stratégies municipales et logiques de protection de l'espace en milieu périurbain : le cas de l'aire urbaine de Paris », in MOLINERO HERNANDO F., Espacios naturales protegidos, Baeza : AGE, Universidad Internacional de Andalucia, 373-384.

GRISON J.-B., 2009, La très petite commune en France : héritage sans avenir ou modèle original, thèse de géographie, Clermont-Ferrand : Université Blaise-Pascal, 406 p.

KAYSER B., 1990, La renaissance rurale : sociologie des campagnes du monde occidental, Paris : Armand Colin, $320 \mathrm{p}$.

PERRIER-CORNET P. (dir.), 2002, Repenser les campagnes, Paris : DATAR, La Tourd'Aigues : éditions de l'Aube, 288 p.

ROLLAND-MAY C., 2001, «Périphéries, bordures, marges territoriales : sous les mots, les concepts », in Regards croisés sur les territoires de marge(s), Strasbourg: Presses universitaires de Strasbourg, 39-60. 